Nouvelles perspectives en sciences sociales

Revue internationale de systémique complexe et d'études relationnelles

\title{
Les Arts au service de l'identité : le rôle des rapports de comités d'enquête dans la définition de l'art minoritaire
}

\section{Lucie Hotte}

Volume 8, numéro 2, mai 2013

Sur le thème de représentations identitaires et expressions culturelles de la francophonie canadienne à travers ses pratiques artistiques et médiatiques

URI : https://id.erudit.org/iderudit/1016472ar

DOI : https://doi.org/10.7202/1016472ar

Aller au sommaire du numéro

Éditeur(s)

Prise de parole

ISSN

1712-8307 (imprimé)

1918-7475 (numérique)

Découvrir la revue

Citer cet article

Hotte, L. (2013). Les Arts au service de l'identité : le rôle des rapports de comités d'enquête dans la définition de l'art minoritaire. Nouvelles perspectives en sciences sociales, 8(2), 101-117. https://doi.org/10.7202/1016472ar

\section{Résumé de l'article}

Cet article porte sur les attentes des élites franco-canadiennes, et plus particulièrement franco-ontariennes, à l'égard des artistes et de leurs oeuvres dans le contexte des transformations sociétales qui ont cours durant les années 1960 et 1970. Il analyse plus précisément les trois rapports d'enquêtes commandés par le gouvernement ontarien entre 1969 et 1991, soit ceux du Comité franco-ontarien d'enquête culturelle (communément appelé le Rapport Saint-Denis, déposé en 1969), du Groupe d'étude sur les arts dans la vie franco-ontarienne, intitulé Cultiver sa différence. Rapport sur les arts dans la vie franco-ontarienne (ou le Rapport Savard, déposé en 1977) et du Groupe de travail pour une politique culturelle des francophones de l'Ontario, dont le titre est RSVP. Clefs en main (ou le Rapport Grisé, déposé en 1991). L’analyse permet de cerner les divers rôles, le plus souvent de cohésion sociale et de construction identitaire, que les élites souhaitent faire jouer aux artistes et aux oeuvres et de dégager les principaux enjeux et défis que les conceptions de l'art proposées, voire imposées, posent aux artistes. 


\section{Les Arts au service de l'identité: le rôle des rapports de comités d'enquête dans la définition de l'art minoritaire}

Lucie HotTe

Chaire de recherche sur les cultures et les littératures francophones du Canada, Ottawa

\section{Introduction}

T es rapports entre les instances politiques, les idéologies dominantes et les pratiques artistiques sont fort complexes. En ce qui a trait à la littérature, dont il sera plus particulièrement question ici, il est toutefois possible de les définir en fonction de deux types de liens. La première catégorie regroupe les liens que j'appellerai " représentationnels ». En effet, la littérature, grâce à sa capacité de représenter des univers possibles, peut mettre en scène le politique, incarner les idéologies à travers les croyances et les actions des personnages qui les habitent, ou décrire, voire critiquer, une société donnée et les jeux de pouvoirs qui y ont cours. Le deuxième type de liens touche à des questions institutionnelles. Il découle du fait que la littérature est elle-même déterminée par le politique - voire la politique - et les idéologies dominantes puisqu'elle est un phénomène social dont la pratique est à la fois régie par des politiques (subventions aux auteurs, aux éditeurs, consécration par des prix nationaux, enseignement...) 
et orientée par des croyances idéologiques quant à ses fonctions dans la société. C'est ce deuxième type de liens unissant politique, idéologies et littérature, plus particulièrement dans le contexte des communautés francophones minoritaires du Canada, que j'aimerais explorer ici ${ }^{1}$.

À la fin des années 1960, surviennent diverses transformations sociétales qui auront des répercussions majeures sur la pratique de la littérature en contexte minoritaire francophone au Canada. Je vais d'abord revenir brièvement sur les éléments contextuels qui sont au fondement d'une redéfinition de la place des arts et de la littérature dans les communautés francophones minoritaires du Canada pour ensuite me pencher sur la façon dont les " élites " franco-ontariennes ont défini le rôle des arts et des artistes à partir d'une analyse des trois rapports d'enquêtes commandés par le gouvernement ontarien entre 1969 et 1991, seul gouvernement provincial, à l'extérieur du Québec, à le faire, soit ceux du Comité franco-ontarien d'enquête culturelle (communément appelé le Rapport Saint-Denis, déposé en 1969), du Groupe d'étude sur les arts dans la vie franco-ontarienne, intitulé Cultiver sa différence. Rapport sur les arts dans la vie franco-ontarienne (ou le Rapport Savard, déposé en 1977) et du Groupe de travail pour une politique culturelle des francophones de l'Ontario, dont le titre est RSVP. Clefs en main (ou le Rapport Grisé, déposé en 1991). Enfin, dans un troisième temps, je chercherai à cerner les enjeux principaux et les défis que les conceptions de l'art proposées par les élites posent aux artistes.

\section{Le contexte sociohistorique}

Les années 1970 sont le moment d'une affirmation identitaire francophone sans précédent au Canada. De nouvelles identités s'élaborent suite à la scission du Canada français découlant de divers facteurs sociaux. Trois d'entre eux auront un effet déterminant. D'abord, la modernisation de l'État entraîne un recul

$1 \quad$ Voir aussi mon article « Artiste, animateur culturel ou médiateur culturel? Le rôle des artistes dans les communautés francophones du Canada ", Minorités linguistiques et Société, La Francophonie canadienne en mouvement : continuité ou rupture?, $n^{\circ} 3$, printemps 2013, à paraître. 
de la religion et du rôle des congrégations religieuses dans la société, dans les domaines de l'éducation, de la santé et des services caritatifs. Les structures traditionnelles cèdent le pas à un état-Providence qui fonctionne essentiellement en anglais. Dans sa relation avec le monde, le francophone minoritaire ne se définit donc plus en lien étroit avec sa paroisse et sa religion mais en relation avec le territoire provincial ou national, où il habite et où il doit nécessairement interagir avec l'autre majoritaire, anglophone, pour l'obtention de services. Cette transformation va de pair avec la popularité de la contre-culture chez la jeunesse francophone, qui se sent davantage liée aux jeunes occidentaux de partout dans le monde, qu'aux générations qui les précèdent dans leur propre collectivité. Larrivée de plusieurs médias de masse (la télévision, surtout, mais aussi le cinéma, la musique populaire, les revues) dans les villages les plus éloignés et même dans l'intimité des maisons contribuent à la diffusion de ces nouvelles façons de penser le monde. Ils servent également à la diffusion de formes artistiques nouvelles, différentes de celles produites traditionnellement par la communauté francophone. Cette ouverture à l'autre influencera de toute évidence la production des jeunes artistes francophones du Canada qui s'inspireront de modèles venus d'ailleurs. Le théâtre d'André Paiement en Ontario, la poésie de Gérald Leblanc en Acadie ou encore l'écriture de J. R. Léveillé au Manitoba prennent forme au contact de la culture américaine, ou encore, du nouveau roman français. Souvent, les jeunes artistes, surtout dans le domaine de la chanson, allieront folklore et formes artistiques modernes. L'identité déborde alors les frontières territoriales et épouse plutôt des limites générationnelles : les jeunes s'opposent aux vieux autant sinon plus que les anglophones aux francophones. Enfin, et c'est là sans doute l'élément le plus déterminant car le plus politique, la montée du nationalisme au Québec qui amène les Québécois à s'identifier en fonction de leur territoire provincial et à délaisser l'appellation "Canadiens français ", pousse les francophones vivant à l'extérieur de la Belle province, qui se veut désormais un 
pays, à se trouver aussi de nouvelles identités. Les jeunes surtout, qui prennent la parole à cette époque, seront interpellés par cette quête identitaire. À Sudbury, par exemple, les institutions littéraires et artistiques qui voient le jour à cette époque - la maison d'éditions Prise de parole, le Théâtre du Nouvel-Ontario, la Galerie du Nouvel-Ontario, le centre culturel La Slague, la Nuit sur l'étang - sont fondées dans le but de permettre l'expression d'une identité proprement franco-ontarienne qui prendra forme dans des œuvres créées par des Franco-Ontariens, pour des Franco-Ontariens, se déroulant en Ontario français et racontant la réalité de la communauté.

Ainsi, tous ces facteurs et notamment les acerbes critiques des Canadiens français du Québec à l'égard de la place dévolue aux francophones au Canada et le désir de plusieurs d'entre eux de militer pour un Québec indépendant, incitent le gouvernement fédéral à mettre sur pied la Commission royale d'enquête sur le bilinguisme et le biculturalisme qui conduira à la promulgation de la Loi sur les langues officielles, en 1969, et à la création des divers programmes de subvention des arts et de la culture administrés par le Secrétariat d'État, nouvellement créé. Deux éléments concourent donc à transformer la conception du rôle de l'artiste en contexte minoritaire, si ce n'est les pratiques artistiques elles-mêmes, au cours des années 1970. D’abord, l'affirmation identitaire néo-nationaliste et contre-culturelle qui prend forme dans les diverses communautés francophones et influe sur la structure même des œuvres produites, en deuxième lieu, les politiques publiques concernant le financement des arts qui promeuvent une conception de l'art dans les critères utilisés pour juger des demandes de subventions aux artistes, comme l'illustre l'étude de Marion Denizot portant sur "plus d'un siècle d'histoire de l'intervention publique en matière artistique ${ }^{2}$ " en France. Les élites des communautés francophones en contexte minoritaire vont aussi vouloir instrumentaliser les artistes de leurs

2 Marion Denizot, « Du théâtre populaire à la médiation culturelle : autonomie de l'artiste et instrumentalisation ", Lien social et Politiques, n 60, 2008, p. 64. 
communautés et leur production artistique afin de promouvoir et valoriser une nouvelle identité liée au territoire provincial.

\section{Les comités d'enquête et le rôle dévolu aux artistes}

En Ontario, trois comités ou groupes d'enquête - ceux déjà présentés ci-haut - seront mis sur pied afin d'étudier la situation des arts et de la culture francophones dans la province. Ces comités témoignent du rôle que les élites (qui y siègent et qui ont souvent tiré les ficelles afin que soient créés ces groupes d'enquête) croient que les artistes et écrivains devraient jouer dans la communauté, voire ceux qu'ils aimeraient leur faire jouer. En effet, les rapports qui sont déposés au gouvernement ainsi que les listes de recommandations qui devraient, selon eux, être mises en place pour assurer la qualité de vie des Franco-Ontariens, si ce n'est leur survie, témoignent de l'idéologie qui préside à leur élaboration et surtout de la conception que se font les membres des commissions de l'art et de l'artiste.

Le Comité franco-ontarien d'enquête culturelle, le premier à être mis sur pied, reçoit comme mandat ${ }^{3}$, en mai 1967, de « faire le point sur la participation des Franco-Ontariens à la vie artistique et culturelle de cette province ${ }^{4}$. Les trois bases sur lesquels s'appuient leur réflexion, énoncées dès le début du rapport, illustrent bien les présupposés unissant langue, culture et pratiques artistiques qui orienteront les recommandations du comité. D'abord, le comité postule l'existence d'une culture canadiennefrançaise distincte, énoncé communément admis, pour ensuite soutenir que les Franco-Ontariens se rattachent à cette culture. Étant donné le caractère distinct de cette culture francophone en

3 Bien que soumis au coordonateur de la division d'Échanges cultuels du ministère de l'Éducation de l'Ontario, le rapport soutient cependant que c'est le Premier ministre de l'Ontario qui lui a confié ce mandat. Le Rapport Savard rapporte cependant que ce sont " des leaders franco-ontariens regroupés autour de M. Roger Saint-Denis [qui] obtiennent l'aide financière du gouvernement provincial pour mener une enquête sur la vie culturelle des Franco-Ontariens. " Pierre Savard, Rhéal Beauchamp et Paul Thompson, Cultiver sa différence. Rapport sur les arts dans la vie franco-ontarienne, septembre 1977, p. 30.

$4 \quad$ Ibid., p. 9. 
Ontario et afin de lui redonner "vigueur et santé ", il faut promouvoir la "vie artistique des Franco-Ontariens, émanation d'une culture propre [...] par des solutions conformes à ses fondements culturels ${ }^{5} »$. Ces constats posés d'emblée, dès l'introduction du rapport, attestent du présupposé qui sous-tend les travaux du Comité et ses réflexions. Celui-ci est ancien et date $\mathrm{du} \mathrm{XVI}^{\mathrm{e}}$ siècle $^{6}$. En effet, au moment où naissent les Étatsnations, où sont valorisées les langues vulgaires qui deviennent les langues nationales, les arts et plus particulièrement la littérature, sont perçus comme "l'expression privilégiée d'une culture ", expression qui revient constamment tant dans les documents gouvernementaux - tels les rapports du Commissaire aux langues officielles - que dans les discours des sociologues, politicologues et autres chercheurs en sciences sociales. Ainsi, langue, culture et arts, auxquels se joint bien souvent le concept d'identité, se trouvent intrinsèquement liés dans l'imaginaire collectif. Il n'est dès lors pas étonnant que le Comité franco-ontarien d'enquête culturelle adopte la même définition de la culture que la Commission royale d'enquête sur le bilinguisme et le biculturalisme, définition qui souligne aussi le lien entre culture et langue : " la culture est une manière globale d'être, de penser, de sentir; c'est un ensemble de mours et d'habitudes, c'est aussi une expérience commune; c'est, enfin, un dynamisme propre à un groupe qu' unit une même langue ${ }^{7}$ ". Aussi, lorsque les membres du comité se tournent vers les pratiques artistiques, ils choisissent d'utiliser le terme, peu fréquent, d'» art situé ". En effet, ce syntagme permet de souligner une conception de l'art ancré dans un milieu, donc situé. La définition qu'en donne le rapport est à mon avis très révélatrice :

\section{$5 \quad$ Ibid., p. 14.}

6 Voir à ce sujet, l'excellent article synthèse d'Itamar Evan-Zohar, «The Role of Literature in the Making of the Nations of Europe: a Socio-Semiotic Study", Applied Semiotics/Sémiotique appliqué, vol. 1, n 1, 1996, p. 39-59. Voir aussi Pascale Casanova, La République mondiale des lettres, éd. revue et corrigée, Paris, Seuil, coll. "Points », 2008 [1999].

7 Canada. Commission royale d'enquête sur le bilinguisme et le biculturalisme, Les Langues officielles, Ottawa, Imprimeur de la reine, 1967, p. XXI. 
L'art « situé » identifie les manifestations, les expressions concrètes de ce style de vie et de pensée que définit la culture. Dans notre optique, l'art constitue l'expression, dans des formes esthétiques, de la réalité et des caractéristiques d'une culture donnée. S'il est des "formes universelles" (la peinture, la musique, la sculpture, etc.) à la disposition de l'esprit créateur, il ne demeure pas moins que l'artiste utilise ces cadres pour traduire un sentiment, une réalité perçue, une expression fugitive de beauté selon des conventions et à travers un prisme unique, propre à un groupe défini culturellement ${ }^{8}$.

Les membres du comité ne manquent pas de souligner que cette conception de l'art va à l'encontre de celle adoptée par « les tenants d'une cité fraternelle où l'art abolit les frontières et amenuise les antagonismes ${ }^{9}$ ». Ils enchaînent en présentant une série d'exemples d'écoles artistiques, telle l'école florentine en peinture, qui, selon eux, prouvent le lien entre le génie propre à une communauté et les formes artistiques pratiquées par ses artistes. Il est pour eux « impossible d'imaginer un art qui ne soit pas "situé" culturellement ${ }^{10}$ ». En effet, ils croient fermement que l'art ne s'incarne que dans une culture et réitèrent à maintes reprises " la nécessaire dépendance de l'art - sous toutes ses formes -, à l'égard d'une culture nourricière, inspiratrice ${ }^{11}$ ». Or, ce postulat sera rapidement inversé puisque finalement ce sera la présence de productions artistiques nombreuses, valorisant la réalité et l'identité du groupe, qui constituera un indice de la vitalité de la culture et non l'inverse : plus une communauté comptera d'artistes, plus elle sera considérée vivante. Ce premier rapport, comme les suivants, nous le verrons à l'instant, privilégie ainsi une des deux représentations principales de l'artiste, soit celle de l'artiste engagé, à qui échoit une fonction sociale, au détriment de celle de l'artiste créateur, qui est préoccupé par des questions proprement esthétiques. L'art et les artistes se trouvent conséquemment instrumentalisés : ils doivent servir une cause.

Le rapport du Groupe d'étude sur les arts dans la vie francoontarienne, Cultiver sa différence. Rapport sur les arts dans la vie

\begin{tabular}{ll}
\hline 8 & Ibid., p. 19. Je souligne. \\
9 & Ibid. \\
10 & Ibid. \\
11 & Ibid., p. 20.
\end{tabular}


franco-ontarienne, s'élabore sur des présupposés similaires. Sa définition de la culture, inspirée aussi de celle du Rapport de la Commission royale d'enquête sur le bilinguisme et le biculturalisme (1967) est fondamentalement la même et mise sur le même rapport entre langue, culture et pratiques artistiques. Le fait que les deux rapports commencent avec un portrait " de la vie culturelle et artistique ${ }^{12}$ " qui est essentiellement une description de la situation démographique, économique et politique des Franco-Ontariens en dit long sur l'appareil conceptuel de l'enquête : la situation des francophones en Ontario est déplorable, l'assimilation fait des ravages, une revalorisation de la culture par les arts permettrait de contrer ce déclin du fait français en Ontario. On peut certes arguer que les commissaires et autres membres de ces groupes d'enquête sont motivés d'abord par un désir de promouvoir les arts en Ontario et d'inciter le gouvernement à fournir aux artistes les moyens de créer et diffuser leurs productions artistiques en utilisant des arguments « sociaux " que le gouvernement est apte à comprendre. Je pense cependant que les commissaires énonçaient aussi leur profonde conviction que l'art en contexte minoritaire doit d'abord et avant tout servir.

Il convient cependant de mentionner que le Rapport Savard est plus nuancé que celui du comité présidé par Roger SaintDenis. En effet, on y énonce cinq conceptions différentes de l'art, proposées par les diverses et nombreuses personnes rencontrées au cours de l'enquête, qui soulignent soit : 1) la valeur esthétique de l'art, 2) sa valeur éducative, 3) son rôle en tant que divertissement 4) sa fonction sociale voire 5) son absence totale de fonction, l'art étant alors considéré comme un "opium " telle la religion pour Marx. Même si le rapport soutient que toutes ces conceptions sont valables, il n'en demeure pas moins que de l'avis des membres du groupe, "il est indispensable que l'Ontario français s'inspire des formes tant présentes que passées de la culture française et québécoise. Mais il doit le faire à travers l'expérience ontarienne et non d'une façon détachée de tout

$\overline{12}$ Ibid., p. 31. Dans le Rapport Savard, le chapitre s’intitule «Société, culture et arts ». 
support socio-culturel ${ }^{13}$. » Ainsi pour eux, "Les arts sont l'expression, dans des matériaux et selon des techniques, d'une saisie du réel [...] codifiée originairement par la langue ${ }^{14}$ ». Or, si le Groupe nuance, tout au long du rapport sa conviction profonde, c'est à cause du discours prôné par les artistes qui déplorent cet état de fait :

il ne faut pas conclure à une relation inévitable entre les arts et la lutte pour la survie de la langue. Trop de gens se méprennent à ce sujet, comme l'ont déploré devant nous maints artistes franco-ontariens : le thème de la survivance est tellement obsédant dans la collectivité francoontarienne, du moins dans ses organismes de défense et de promotion de la langue et dans les écoles, que si une expression artistique ne véhicule pas d'éléments clairement identifiables à ces luttes de survivance et aux conflits avec les autres, elle n'est pas perçue comme francoontarienne $e^{15}$.

Les enquêteurs soutiennent donc que " [l]'expression artistique franco-ontarienne ne peut se nourrir exclusivement du thème de la survie culturelle et linguistique : elle doit aussi s'inspirer des autres grands problèmes de notre temps ${ }^{16}$ ", mais ils modulent aussitôt : "Bien entendu, le Franco-Ontarien, et c'est là son originalité, saura donner à l'expression de problèmes "universels" une tonalité propre qui est la marque de son appartenance à une culture donnée, vécue dans un milieu concret ${ }^{17}$ ». Pour finalement conclure : "En somme, arts et culture sont indissociables. L'art ne peut exister sans la médiation d'une culture située dans un espace et un temps donnés ${ }^{18} "$.

Le troisième rapport paraît 14 ans plus tard, soit en 1991, après la période de revendications identitaires. Propose-t-il pour autant une conception différente de la place de l'artiste et de son rôle dans la société minoritaire? Ce rapport est beaucoup plus succinct que les deux premiers, en grande partie parce qu'il présente en une seule page, située avant la table des matières et

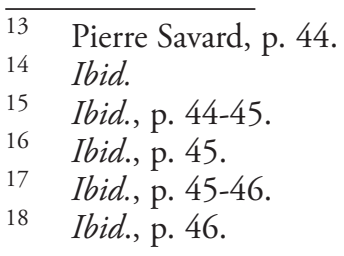


donc avant le rapport lui-même, "Quelques données sur la population franco-ontarienne " alors que les deux autres rapports consacraient de longues pages à cet état des lieux. Le reste du document présente, d'abord, une définition de la culture, puis les sept objectifs qui mèneront en fin de parcours aux stratégies et recommandations proposées, mais pas avant d'avoir fait le point sur la situation actuelle, c'est-à-dire sur les principaux obstacles que doit surmonter la communauté, mais aussi sur ses atouts. Ce rapport propose une définition novatrice de la culture fondées sur cinq plans, 1) humain (les habitudes, les croyances...), 2) temporel (l'histoire), 3) spatial, 4) de l'action (le dynamisme), 5) de la création, qu'il explicite en ces termes : «la culture produit, par ses acteurs culturels dont les artistes sont le fer de lance, des œuvres qui forment et reforment et précisent son identité ${ }^{19}$ ". Ainsi, la production artistique est à nouveau liée consubstantiellement, pourrait-on dire, à l'identité. Enfin, la culture est, selon le Groupe, une émotion fondée sur le sentiment d'» appartenance à la communautée ${ }^{20}$ » et, en dernier lieu, on souligne le lien entre langue et culture en stipulant qu'" [i]l n'y a pas de langue sans culture, comme il n'y a pas de culture sans artistes ${ }^{21} »$. Ce postulat sera réitéré dans la liste des principes directeurs où art, culture et identité seront à nouveau intrinsèquement liés : "L'art est un moyen incontournable de développer la culture. Il est tout à la fois le cadre indispensable à la culture et la somme de ses manifestations. Si l'art n'est pas toute la culture, il en est le point ultime d'expression ${ }^{22}$ ». Ou encore "L'artiste est le ferment de la culture $^{23}$ ». Ces deux positions apparaissent contradictoires : l'art représente-il la culture qui existe indépendamment de lui ou l'art fonde-t-il une culture qui ne saurait exister sans lui? Là est sans doute la question. Ce rapport souligne cependant qu'il ne faut

19 Groupe de travail pour une politique culturelle des francophones de l'Ontario, RSVP! Clefs en main, Toronto, [s.é.], 1991, p. 9.

20 Ibid.

21 Ibid.

22 Ibid., p. 10.

23 Ibid. 
pas «prendre la langue pour la culture ${ }^{24}$ ", ce qui lui permet d'énoncer son premier objectif, soit de "fonder l'identité sur la culture et non sur la langue ${ }^{25}$ ». Pourtant, nombre d'affirmations dans le rapport semblent toujours entériner l'ancien couple culture et langue. Les stratégies proposées misent, dans une large mesure, sur ce lien fondamental qui définit le rôle de l'artiste en contexte minoritaire. On peut en fait se demander si ce ne sont pas les artistes qui sont perçus comme les mieux placés pour implanter la première stratégie qui vise à « [f] aire de l'animation culturelle un véritable outil de développement " afin de " redonner vie à la culture ${ }^{26}$ ». Cela n'est jamais énoncé de façon explicite dans le rapport, il s'agit là cependant d'un mandat qui est fréquemment attribué aux artistes en contexte minoritaire et que ceux-ci acceptent parfois de remplir, mais qu'ils dénoncent le plus souvent ${ }^{27}$.

\section{Principaux enjeux : l'artiste, un animateur social? Un médiateur culturel?}

Le lien entre art et culture est sans contredit l'élément problématique de l'équation. Ce n'est pas parce qu'on le présente comme inaltérable qu'il devient problématique, mais bien parce qu'il fonde certains présupposés par rapport à l'art en contexte minoritaire et au rôle des artistes. Si l'art minoritaire est bien souvent perçu comme un art $d u$ minoritaire comme le dit si bien Jean Lafontant ${ }^{28}$, c'est-à-dire un art qui représente la réalité de la communauté minoritaire, l'artiste lui devient dès lors soit un

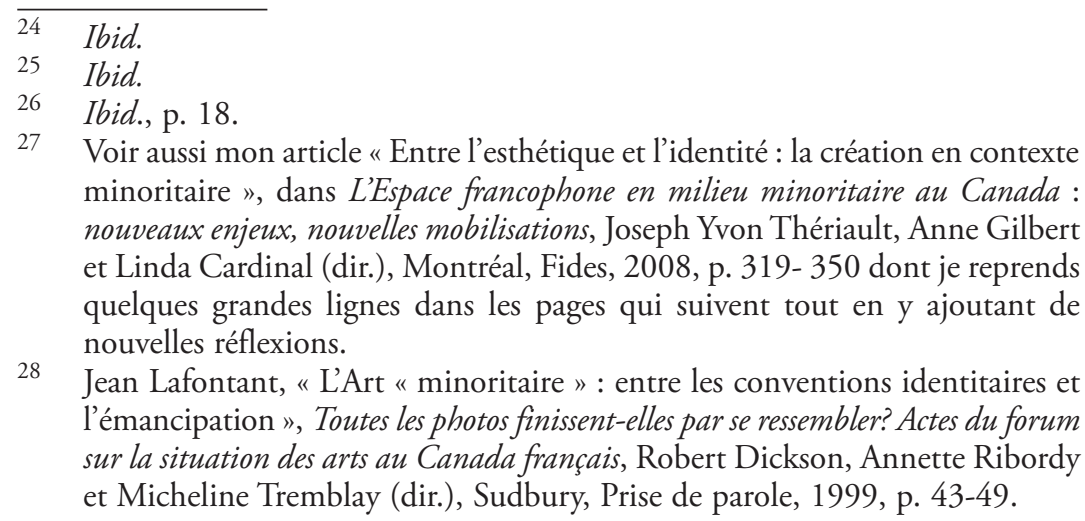


" animateur social ", soit un médiateur culturel. En effet, comme le souligne Marion Denizot, dans certains contextes ${ }^{29}$, dont celui où se produit une

redéfinition du lien social, le pouvoir politique est tenté d'appeler à une mobilisation accrue de l'artiste sur le terrain de la médiation culturelle, au risque de minorer la dimension proprement artistique de sa démarche, fondée sur le geste créateur irréductible d'un individu ou d'un collectif, et de réduire, par conséquent, son autonomie artistique, c'est-à-dire sa capacité à créer en fonction de normes et de valeurs propres au champ artistique $^{30}$.

C'est évidemment ce qui se passe en Ontario français, notamment durant les années 1970 et 1980, où le désir de "refonder le lien social ", de " refaire société », pour utiliser dans une forme modifiée l'expression de Joseph-Yvon Thériault, pousse les acteurs sociaux à mobiliser les arts et à les mettre au service de la communauté et de l'affirmation identitaire, et transforme l'artiste en chantre du groupe. C'est ce qui a poussé Herménégilde Chiasson à affirmer, ironiquement, que

L'art en milieu minoritaire [...] se manifeste surtout sous la forme de chronique, d'illustration ou d'archives. Il faut documenter le vécu de la collectivité, s'assurer qu'elle survivra dans les artefacts. [...] On veut voir et entendre la vérité. [...] Le réalisme donc et, si possible, l'hyperréalisme. [...] On veut du théâtre franco-ontarien, de la poésie acadienne ou de la musique franco-manitobaine. En deuxième lieu, on veut que cette forme d'art nous identifie, donc nous illustre, nous archivise ${ }^{31}$.

Il dénonce cet état de fait lorsqu'il s'exclame : «Nous sommes devenus des Acadiens avant d'être des artistes. L'adjectif a supplanté le nom ». En effet, lorsque les intervenants sociaux instrumentalisent l'artiste, ils "radicalis[ent] l'opposition entre légitimité artistique et légitimité sociale ${ }^{32} »$. L'artiste se trouve dès lors pris dans un dilemme : soit il pratique son art en fonction

$29 \quad$ Marion Denizot mentionne aussi les contextes de contrainte budgétaire et de tensions sociales.

30 Marion Denizot, loc. cit., p. 64. Je souligne. Denizot s'inspire et renvoie à Pierre Bourdieu, Les Règles de l'art. Genèse et structure du champ littéraire, Paris, Seuil, 1992.

31 Herménégilde Chiasson, «Toutes les photos... », Toutes les photos finissent-elles par se ressembler, 1999, op. cit., p. 86-87.

32 Marion Denizot, loc. cit., p. 64. 
des normes qui lui sont propres et cherche à atteindre l'excellence dans sa discipline qui lui permette de s'y faire un nom, soit il se plie aux demandes de la collectivité, agit comme médiateur culturel afin de "démocratiser " les arts et de valider l'identité collective, et produit donc de l'art engagé, le plus souvent connoté négativement dans le milieu artistique, et voit ses chances de renommée diminuées. L'artiste minoritaire doit donc trouver un équilibre, toujours précaire, entre sa vocation d'artiste et son appartenance à la collectivité. Henri-Dominique Paratte pose la question en termes clairs : "Comment être, d'une certaine manière, le porte-parole du groupe, sans être étouffé par lui et sans sacrifier la liberté fondamentale qui est le point de départ de toute création artistique et son utilité profonde pour l'évolution du groupe? ?3 $^{33}$

Selon Marion Denizot, l'instrumentalisation de l'artiste comme la survalorisation de son rôle en tant que médiateur culturel seraient " deux symptômes d'un phénomène plus général : la substitution de la culture à l'art ${ }^{34}$ ". Elle cite à cet égard le philosophe spécialiste en esthétique, Marc Jimenez qui soutient qu'» [à] l'intérieur de la sphère culturelle, là où l'omnipotence du culturel s'impose au détriment de l'esthétique, tout doit être lisse, dépourvu d'équivoques et d'ambiguïtés ${ }^{35} \%$. L'artiste devient dès lors « un simple pourvoyeur d'objets culturels ${ }^{36}$ ». Je ne peux que reprendre ici la conclusion à laquelle en vient Denizot : « au nom de la cohésion sociale à (re)construire, le "trop" de médiation conduit à léser l'objet même de celle-ci : l'œuvre. La seule réponse serait alors de maintenir le projet artistique au cœur du processus de médiation et d'affirmer son primat sur tout objectif externe

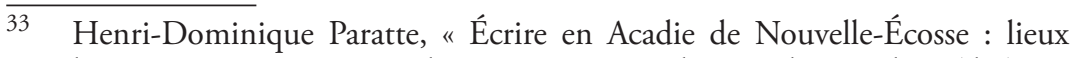
littéraires et création en milieu minoritaire », dans André Fauchon (dir.), La production culturelle en milieu minoritaire, Winnipeg, Presses universitaires de Saint-Boniface, 1994, p. 194.

34 Marion Denizot, op. cit., p. 73.

35 Ibid. Marion Denizot cite Marc Jimenez, La Critique. Crise de l'art ou consensus culturel?, Paris, Klincksieck, 1995, p. 37.

36 Marion Denizot, op. cit., p. 73: "L'artiste ne serait-il pas devenu un simple pourvoyeur d'objets culturels, tel que le dénonçait déjà Hannah Arendt? ". 
au champ artistique ${ }^{37}$ ». Mais cette position est-elle viable en contexte minoritaire?

$\mathrm{Si}$, dans un premier temps, soit durant les années 1970 et au début des années 1980, les artistes ont accueilli avec joie l'intérêt dont faisaient preuve les instances politiques et les élites à leur égard, notamment lorsque cet intérêt conduisait au financement d'activités artistiques et culturelles, ils sont devenus rapidement plus critiques face à ces politiques subventionnaires lorsqu'ils ont compris que celles-ci limitaient en fait leur pratique artistique. L'idéologie contre-culturelle et marxiste qui les anime durant les années 1970 et au début des années 1980 fait en sorte que les artistes eux-mêmes perçoivent leur rôle en société comme celui d'un éclaireur de conscience, d'un mobilisateur ou encore d'un animateur culturel. Cependant, les artistes se sentent rapidement brimés par ces contraintes idéologiques qui pèsent sur leur art. En effet, alors que les élites, tel la Fédération culturelle canadienne-française, soutiennent encore aujourd'hui que "[p]our un avenir garant d'une forte vitalité de nos communautés, il faut remettre en perspective la force des arts et de la culture à titre d'agent rassembleur ${ }^{38}$ ", les artistes eux refusent à présent d'être des animateurs sociaux voire de devenir des médiateurs culturels.

La prégnance des diktats identitaires place l'artiste face à un dilemme : soit il refuse d'ancrer son ouvre dans la réalité ambiante et se voit priver de sources de financement, de lieux de diffusion (dans les écoles, par exemple) ou de promotion dans les journaux et au sein de la communauté, soit il se plie aux attentes communautaires et se sent limité dans les formes qu'il peut utiliser et les thèmes qu'il peut aborder dans ses œuvres. En fait, le discours social oblige l'artiste à choisir entre deux représentations de l'artiste, soit « l'artiste créateur, au service d'enjeux proprement esthétiques, et l'artiste engagé, acteur d'une fonction sociale de l'art ${ }^{39} »$. Certes, certains artistes pratiquent avec plaisir

$37 \quad$ Ibid.

38 Fédération culturelle canadienne-française, Imagine, Ottawa, [s.é.], 2007, p. 8. Disponible en ligne sur le site de la FCFA : http://www.fcfa.ca/documents/545.pdf. Consulté le 19 juillet 2010. 
et volontairement un art "situé ». Certes, d'autres artistes pratiquent avec succès un art universaliste, même en contexte minoritaire. Il n'en demeure pas moins que concevoir l'art produit en contexte minoritaire en fonction de critères sociologiques, idéologiques et politiques, c'est placer ces pratiques artistiques dans une catégorie distincte de celle où sont logées les pratiques artistiques majoritaires. C'est donc minoriser davantage l'artiste minoritaire en le plaçant dans une catégorie à part de ses confrères et consœurs œuvrant en contexte majoritaire.

\section{Conclusion}

Le rôle des commissions d'enquête sur les arts et la culture en Ontario français est donc à la fois positif et négatif. D'une part, les commissions ont souligné l'importance, pour le développement des arts et la survie de la communauté francophone, d'investir dans les arts, de promouvoir la culture et de se doter d'institutions permettant d'en assurer la vitalité. D'autre part, la définition trop restrictive de l'art que les membres des comités adoptent nuit au développement des artistes qui se sentent coincés dans une conception sociologique et engagée de l'art. Y a-t-il une solution? Celle-ci consisterait, à mon avis, à arriver à concilier les exigences artistiques au désir de la communauté. Mais, pour que cela soit possible, il faut que la communauté soit consciente que les œuvres n'ont pas à promouvoir explicitement la culture locale, n'ont pas à mettre en scène la réalité régionale ou provinciale, n'ont pas à peindre les mœurs et les coutumes du groupe pour qu'elles participent à la vitalité de la collectivité. En effet, le simple fait de produire des ouvres en contexte minoritaire - et d'autant plus lorsque ces œuvres peuvent se mesurer à celles produites en contexte majoritaire parce quelles correspondent aux normes esthétiques qui y sont en vigueur - contribue à valoriser la collectivité, incite les jeunes à devenir à leur tour créateurs, dans leur langue maternelle, et encourage la population en général à apprécier ces œuvres d'art produites chez eux, par les leurs dans leur langue, car celles-ci sont d'aussi grande qualité que celles venues d'ailleurs. Ce n'est donc pas seulement 
l'art particulariste qui a un effet bénéfique pour la communauté, l'art décontextualisé contribue tout autant au bien-être d'une communauté. En fait, je crois fermement que ce sont même les œuvres les moins engagées qui risquent d'être les mieux reçues, les mieux perçues et les plus encensées par le public puisque celui-ci cherche le plus souvent à se divertir lorsqu'il consomme des produits artistiques et non pas à se faire endoctriner. Trouver ce juste milieu constitue, selon moi, un beau défi que nous sommes appelés à relever.

\section{Bibliographie}

Bourdieu, Pierre, Les Règles de l'art. Genèse et structure du champ littéraire, Paris, Seuil, 1992, 480 p.

Canada. Commission royale d'enquête sur le bilinguisme et le biculturalisme, Les Langues officielles, Ottawa, Imprimeur de la reine, 1967, xliii, 229 p.

Casanova, Pascale, La République mondiale des lettres, Paris, Seuil, coll. « Points », 2008 [1999], xvi, 509 p.

Chiasson, Herménégilde, "Toutes les photos... ", dans Robert Dickson, Annette Ribordy et Micheline Tremblay (dir.), Toutes les photos finissent-elles par se ressembler? Actes du forum sur la situation des arts au Canada français, Sudbury, Prise de parole, 1999, p. 84-91.

Denizot, Marion, « Du théâtre populaire à la médiation culturelle : autonomie de l'artiste et instrumentalisation ", Lien social et Politiques, $\mathrm{n}^{\circ}$ 60, 2008, p. 63-74.

Groupe de travail pour une politique culturelle des francophones de I'Ontario, RSVP! Clefs en main, Toronto, [s.é.], 1991, 64, 66 p.

Hotte, Lucie « Artiste, animateur culturel ou médiateur culturel? Le rôle des artistes dans les communautés francophones du Canada ", Minorités linguistiques et Société, $\mathrm{n}^{\circ} 3$, printemps 2013, à paraittre. 
Hotte, Lucie, "Entre l'esthétique et l'identité : la création en contexte minoritaire ", dans Joseph Yvon Thériault, Anne Gilbert et Linda Cardinal (dir.), L'Espace francophone en milieu minoritaire au Canada: nouveaux enjeux, nouvelles mobilisations, Montréal, Fides, 2008, p. 319350.

Itamar, Evan-Zohar, "The Role of Literature in the Making of the Nations of Europe: a Socio-Semiotic Study», Applied Semiotics/Sémiotique appliquée, vol. 1, n 1, 1996, p. 39-59.

Lafontant, Jean, "L'Art " minoritaire " : entre les conventions identitaires et l'émancipation ", Robert Dickson, Annette Ribordy et Micheline Tremblay (dir.), Toutes les photos finissent-elles par se ressembler? Actes du forum sur la situation des arts au Canada français, Sudbury, Prise de parole, 1999, p. 43-49.

Jiminez, Marc, La Critique. Crise de l'art ou consensus culturel?, Paris, Klincksieck, 1995, 165 p.

Paratte, Henri-Dominique, "Écrire en Acadie de Nouvelle-Écosse : lieux littéraires et création en milieu minoritaire ", dans André Fauchon (dir.), La Production culturelle en milieu minoritaire, Winnipeg, Presses universitaires de Saint-Boniface, 1994, p. 191-230.

Savard, Pierre, Rhéal Beauchamp et Paul Thompson, Cultiver sa différence. Rapport sur les arts dans la vie franco-ontarienne, Ottawa, septembre 1977, xiii, 225 p. 